\title{
Diversidad y distribución de Ganoderma (Polyporales: Ganodermataceae) en Sonora, México
}

\author{
Damian López-Peñ̃a ${ }^{1}$, Aldo Gutiérrez', Eduardo Hernández-Navarro', \\ Ricardo Valenzuela ${ }^{2}$ Y Martín Esqueda ${ }^{1,3}$
}

Botanical Sciences

94 (2): 431-439, 2016

DOI: $10.17129 /$ botsci.463

\section{Resumen}

Se estudiaron siete especies de Ganoderma: G. applanatum, G. curtisii, G. lobatum, G. oerstedii, G. sessile, G. sessiliforme y G. weberianum. De ellas, G. lobatum y G. oerstedii son nuevos registros para Sonora, mientras que G. sessile y G. weberianum para México. El género tiene una amplia distribución en Sonora, en donde se encuentra en bosque de encino, encino-pino, pino-encino, encino abierto e incluso en zonas xerófilas con mezquital. Ganoderma oerstedii se encontró en la base de Stenocereus thurberi, que lo hace el primer registro sobre una cactácea.

Palabras clave: corología, hongos poliporoides, sierra Sonorense, taxonomía.

\section{Diversity and distribution of Ganoderma (Polyporales: Ganodermataceae) from Sonora, Mexico \\ Abstract}

Seven species of Ganoderma: G. applanatum, G. curtisii, G. lobatum, G oerstedii, G. sessile, G. sessiliforme, and G. weberianum are discussed. Ganoderma lobatum and G. oerstedii are new records for Sonora, while G. sessile and G. weberianum for Mexico. The genus has a wide distribution in Sonora, being found in oak, oak-pine, pine-oak, open oak forests, also in xerophytic vegetation like mesquite scrub. Ganoderma oerstedii was founded on Stenocereus thurberi, being its first record on a living Cactaceae.

Key words: chorology, polyporoid fungi, Sonoran sierra, taxonomy.
${ }^{1}$ Centro de Investigación en Alimentación y Desarrollo, A.C. Hermosillo, Sonora, México.

${ }^{2}$ Escuela Nacional de Ciencias Biológicas, Instituto Politécnico Nacional. México, D.F., México.

${ }^{3}$ Autor para la correspondencia: esqueda@ciad.mx 
anoderma P. Karst. (Polyporales: Ganodermataceae) es un género con más de 200 especies descritas, pero muchas son sinónimas (Moncalvo y Ryvarden, 1997; IFP, 2014). Se reconocen dos subgéneros con base en características del píleo (Gottlieb y Wright, 1999a, b): Elfvingia (P. Karst.) Imazeki (especies con superficie del píleo opaca) y Ganoderma (con superficie brillante o laqueada). Por su complejidad y variabilidad, el género ha generado confusiones taxonómicas con determinaciones incorrectas. Estudios recientes indican la importancia del contexto y elementos de la cutícula como caracteres decisivos en la delimitación de las especies (Gottlieb y Wrigth, 1999a, b; Torres-Torres y Guzmán-Dávalos, 2005; Torres-Torres et al., 2012).

En México se han registrado alrededor de 16 especies (Mendoza et al., 2011), de las cuales Ganoderma adspersum, G. applanatum, G. lucidum y G. sessiliforme están citados para Sonora (Esqueda et al., 2010). El género es frecuentemente observado en regiones tropicales, subtropicales y templadas (Gilbertson y Ryvarden, 1986; Zhao, 1989; Torres-Torres et al., 2012). En Sonora se conoce su distribución en la zona urbana de Hermosillo, Reserva Forestal Nacional y Refugio de Fauna Silvestre Ajos-Bavispe (bosque de pino-encino, bosque de galería y matorral desértico micrófilo) y Reserva de la Biosfera Sierra de Álamos-Río Cuchujaqui (selva baja caducifolia) (Esqueda et al., 2010). Sin embargo, existen zonas poco exploradas, como la Sierra de Yécora (bosque de pino-encino y encino-pino), Sierra de Mazatán (bosque de encino, matorral subtropical y mezquital) y Sierra de San Javier (bosque de encino abierto y matorral subtropical), en donde se hizo el presente estudio.

\section{Materiales y métodos}

Se realizaron exploraciones en la Sierra de Yécora (municipio de Yécora), Sierra de San Javier (municipio de San Javier) y Sierra de Mazatán (colindancia de los municipios de Ures, Mazatán y Villa Pesqueira) durante 2012 y 2013, en donde se realizaron 17 recolecciones de Ganoderma. Para el estudio morfológico de los ejemplares se siguieron las metodologías propuestas por Furtado (1965), Largent et al. (1977), Bazzalo y Wrigth (1982), Gottlieb y Wright (1999a, b), Ryvarden (2000) y Torres-Torres et al. (2012). Los principales caracteres macroscópicos estudiados fueron dureza y grosor de la cutícula del píleo, tipo de contexto (homogéneo, relativamente homogéneo o dúplex), presencia de incrustaciones o bandas resinosas y la forma y tamaño de los basidiomas.

La descripción de los colores de los basidiomas se realizó de acuerdo a Kornerup y Wanscher (1978); aunque el código sólo se indica en los nuevos registros para México. Se hicieron preparaciones en solución KOH al 5 \% para la caracterización de estructuras microscópicas. Estas fueron el tamaño y forma de las basidiosporas, disposición de los pilares interparietales de las basidiosporas; forma, tamaño e incrustaciones de los elementos de la cutícula; diámetro de los poros y el grosor del disepimento. También se consideró la reacción de esporas y elementos de la cutícula con el reactivo de Melzer y la presencia de clamidosporas. Se midieron al menos 30 esporas de cada ejemplar, para calcular el coeficiente Q (largo/ancho). Los nombres de las especies y autores están basados en la base de datos Index Fungorum (IFP, 2014). Se generó un mapa de distribución con base en los registros anteriores y el presente trabajo. Los especímenes se depositaron en la colección de hongos "Dr. Martín Esqueda Valle" del herbario de la Universidad Estatal de Sonora (UES).

\section{Taxonomía}

Ganoderma applanatum (Pers.) Pat., Hyménomyc. Eur. (Paris): 143 (1887) (Figura 1A-C). Basidioma de 67-225 × 105-165 × 33-83 mm, perenne, sésil, suberoso a leñoso. Píleo semicircular a ligeramente lobado o irregular; superficie opaca, sulcada concéntricamente, zonada, marrón, en ocasiones cubierta con esporas color óxido. Cutícula de hasta $0.48 \mathrm{~mm}$ de grosor. Elementos de la cutícula de 30-45 × 5.6-10 $\mu \mathrm{m}$, claviformes a digitiformes, en ocasiones con protuberancias laterales y ramificaciones, marrón-rojizo a amarillo-dorado, mezclados con hifas esqueléticas. Basidiosporas de 8-9 × 5.2-6.2 $\mu \mathrm{m}, \mathrm{Q}=1.45-1.53$, elipsoides, marrón claro, exosporio con pilares interparietales libres. 


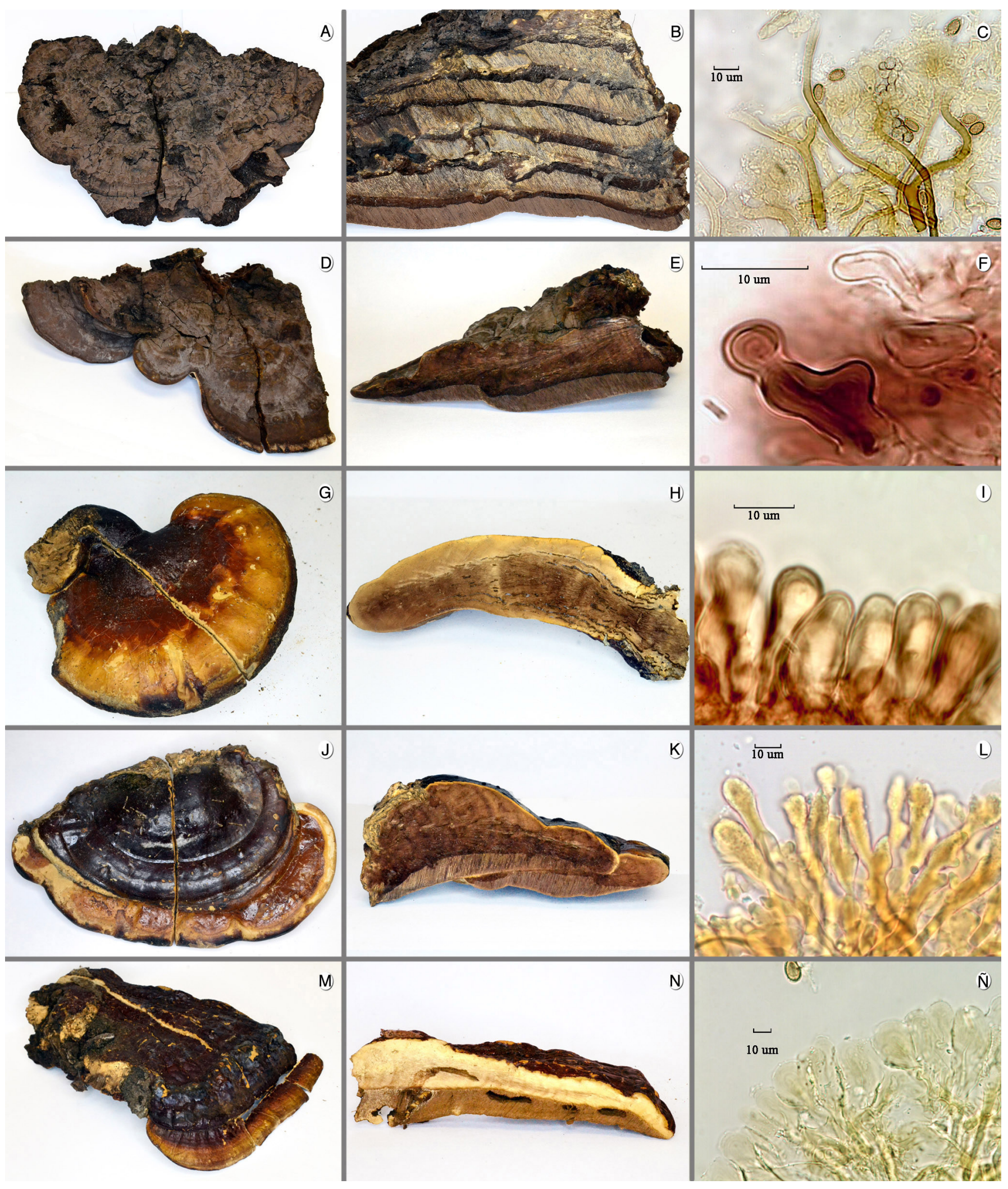

Figura 1. Ganoderma applanatum, G. curtisii, G. lobatum, G. oerstedii y G. sessiliforme. G. applanatum: A) basidioma, B) contexto, C) cutícula; G. lobatum: D) basidioma, E) contexto, F) cutícula; G. curtisii: G) basidioma, H) contexto, I) cutícula; G. oerstedii: J) basidioma, contexto, L) cutícula. G. sessiliforme $\mathbf{M}$ ) basidioma, $\mathbf{N}$ ) contexto, $\tilde{\mathbf{N}}$ ) cutícula. 
Material estudiado. Municipio de Yécora, Sierra de Yécora, E. Hernández, A. Gutiérrez y D. López, 28-09-2012, UES 10420, 10421.

Comentarios. La formación de capas del contexto y de los tubos por las temporadas de desarrollo está acorde con lo descrito para especies perennes. Esta especie puede confundirse con Ganoderma australe (Fr.) Pat., pero se diferencia por la cutícula más delgada de $<0.5 \mathrm{~mm}$ y las basidiosporas más pequeñas de 9-13.5 × 6-10 $\mu \mathrm{m}$ en G. australe. La especie en discusión fue recolectada en bosque de encino-pino sobre la base de Quercus sp.

Ganoderma curtisii (Berk.) Murrill, N. Amer. Fl. (New York) 9(2): 120 (1908) (Figura 1G-I). Basidioma de 22-71 × 17-57 × 10-20 mm, anual, estipitado, suberoso a fibroso. Píleo reniforme a semicircular; superficie brillante, laqueada, carácter que se pierde con el tiempo, quedando irregularmente con zonas opacas; anaranjada a amarilla con tonalidades marrónrojizas. Estípite de $40-54 \times 10-24 \mathrm{~mm}$, lateral, aplanado a cilíndrico, brillante, con tonalidades marrón rojizo. Contexto dúplex, azonado, con 2-4 bandas resinosas, completas o intermitentes a través de todo el contexto. Elementos de la cutícula de 29-47 × 7.3-15.5 $\mu \mathrm{m}$, claviformes, sin incrustaciones, en ocasiones con protuberancias y ramificaciones laterales. Basidiosporas de (7.3-) 7.7-9 $\times 4.5-5.6 \mu \mathrm{m}, \mathrm{Q}=1.44-1.76$, elipsoides a oblongas, con pilares interparietales sublibres.

Material estudiado. Municipio de San Javier, Sierra de San Javier, A. Gutiérrez, E. Hernández, y D. López, 27-09-2012, UES 10402. Municipio de Ures, Sierra de Mazatán, C. Trujillo, E. Hernández, A. Gutiérrez, A. Jiménez y D. López, 18-09-2013, UES 10403.

Comentarios. Ganoderma curtisii es una de las especies con mayor variabilidad morfológica, macro y microscópica dentro del género según Torres-Torres y Guzmán-Dávalos (2005), quienes registraron basidiosporas de (9.2-) 10.4-12.8 (-13.6) × 5.6-8 $\mu \mathrm{m}$ en ejemplares mexicanos de Jalisco, Hidalgo y Morelos, mientras que Zhao (1989) de 8.7-11.3 × 5.2-6.9 (8) $\mu \mathrm{m}$ en ejemplares de China. Steyaert (1980) observó esporas de 8.5-10 (-12) × 5.5-6.2 (-7) $\mu \mathrm{m}$. Lo anterior sugiere que se puede tratar de un complejo de especies. Ganoderma curtisii se recolectó en bosque de encino en una zona conocida como Isla de Montaña, sobre madera muerta de Quercus sp. y en bosque de encino abierto sobre raíz de Quercus sp.

Ganoderma lobatum (Schwein.) G.F. Atk., Annls. mycol. 6(3): 190 (1908) (Figura 1D-F). Basidioma de 36-155 × 55-125 × 26-44 mm, perenne, sésil, en ocasiones subestipitado, suberoso a leñoso. Píleo dimidiado a lobulado, ocasionalmente semicircular; superficie opaca; marrón a marrón-grisáceo. Elementos de la cutícula de 34-40 × 4.4-10 $\mu \mathrm{m}$, claviformes a digitiformes, de pared gruesa, marrón a marrón-rojizo. Basidiosporas de 8.3-9.8 (10.8) × 5.3-6.3 $\mu \mathrm{m}, \mathrm{Q}=1.44-1.81$, elipsoides a oblongas, con pilares interparietales libres.

Material estudiado. Municipio de Yécora, Sierra de Yécora, E. Hernández, A. Gutiérrez y D. López, 28-09-2012, UES 10422, 10423, 10424.

Comentarios. Steyaert (1980) consideró a Ganoderma lobatum dentro del antiguo subgénero Anamixoderma, caracterizado por presentar elementos de la cutícula claviformes, mezclados con terminaciones hifales, lo cual se observó en los ejemplares revisados. Este es el primer registro de G. lobatum para Sonora. Crece en bosque de encino-pino sobre la base de Quercus sp.

Ganoderma oerstedii (Fr.) Torrend, Bull. Torrey bot. Club 29: 606 (1902) (Figura 1J-L).

Basidioma de 105-133 × 85-100 × 28-31 mm, perenne, sésil, fuertemente adherido, leñoso. Píleo flabeliforme-semicircular a irregular; superficie glabra, lisa a irregular, con una capa de laca brillante, la cual puede perderse con el tiempo, sulcada concéntricamente; marrón-rojizo oscuro en casi toda la superficie, con tonalidades anaranjadas hacia el margen. Contexto zonado, con bandas e incrustaciones resinosas conspicuas. Elementos de la cutícula de 40-67.7 × 7.7-12 $\mu \mathrm{m}$, claviformes, con ramificaciones y protuberancias tanto laterales como apicales, amarillo dorado. Basidiosporas de (7.4-) 8.3-10 × 5.1-6.3 (-6.5) $\mu \mathrm{m}, \mathrm{Q}=1.42-1.67$, elipsoides a oblongas, con pilares interparietales parcialmente anastomosados.

Material estudiado. Municipio de Ures, Sierra de Mazatán, A. Gutiérrez, E. Hernández, R. Maldonado y D. López, 10-09-2012, UES 10419.

Comentarios. Las basidiosporas de esta especie varían según la referencia bibliográfica. Ste- 
yaert (1980) registró basidiosporas de 9-10.5 (-12.5) × 6-7.7 (-9) $\mu \mathrm{m}$, mientras que Mendoza et al. (2011) de (10-) 11-13 (-14) × 8-10 $\mu \mathrm{m}$. Estos últimos autores citaron Ganoderma oerstedii de áreas tropicales y subtropicales de Chiapas, Morelos, Sinaloa y Veracruz. Los ejemplares de Sonora provienen de mezquital, en una zona donde se registran temperaturas mayores a $40{ }^{\circ} \mathrm{C}$. Se recolectó sobre la base de Stenocereus thurberi. Éste es el primer registro de la especie desarrollándose sobre una cactácea y la primera cita para Sonora.

Ganoderma sessile Murrill, Bull. Torrey bot. Club. 29: 604. (1902) (Fig. 2A-D).

Basidioma de $50 \times 50 \times 21 \mathrm{~mm}$, anual, sésil, solitario, de consistencia fibrosa a suberosa. Píleo circular-flabeliforme, ligeramente convexo, conchado, dimidiado; superficie laqueada, con una franja delgada hacia el margen sin laca, corrugada, sulcada concéntricamente, cubierta frecuentemente por una fina capa de basidiosporas anaranjado-parduzco (7C4); marrón rojizo (8E8) a marrón oscuro (7F8); margen estéril, agudo, liso, blanquecino en fresco, marrón amarillento (5D8) a marrón (5F8) en seco. Contexto de hasta $12 \mathrm{~mm}$ de grosor, dúplex, zonado, suberoso a esponjoso, con algunos depósitos resinosos dispersos difíciles de observar, la porción superior anaranjado pálido (5A3), la inferior marrón (5F7). Tubos de hasta $9 \mathrm{~mm}$ de longitud, anaranjado tenue (5A4) a anaranjado grisáceo (5B4) cerca de la superficie, ligeramente estratificados. Poros de 4-5 por mm, circulares a angulados, con borde irregular, blancos, se manchan de color marrón al maltratarlos o tocarlos, al igual que todas las especies del género, con una porción marrón (5F7) hacia el margen, de 169-304 $\mu$ m diámetro; disepimento de 21.4-59.6 $\mu \mathrm{m}$ de grosor. Sistema hifal trimítico. Hifas generativas de la trama del contexto no observadas; hifas esqueléticas de 2.2-7.7 $\mu \mathrm{m}$ diámetro, de pared gruesa a sólida, arboriformes y aciculiformes, hialinas a amarillo pálido en la porción superior, marrón pálido a marrón en la porción inferior; hifas conectivas de 1.1-3.1 $\mu \mathrm{m}$ diámetro, fuertemente ramificadas, hialinas. Elementos de la cutícula de 33.7-53 × 8.2-14.9 $\mu \mathrm{m}$, cilíndricos a fuertemente claviformes, ocasionalmente con algunas protuberancias laterales, anaranjado pálido; hifas generativas de 1.8-3.9 $\mu \mathrm{m}$ diámetro, con fíbulas, amarillo pálido; hifas esqueléticas de 1.8-2.8 $\mu \mathrm{m}$ diámetro, con paredes gruesas, marrónamarillento, hifas conectivas de 1.1-2.1 $\mu \mathrm{m}$ diámetro, altamente ramificadas, pared gruesa a sólida, amarillo pálido. Basiodiosporas de 9.6-10.5 × 5.8-6.8 $\mu \mathrm{m}, \mathrm{Q}=1.44-1.72$, elipsoides a oblongas, con ápice truncado, perisporio perforado, exosporio con pilares interparietales sublibres y delgados. Basidios no observados.

Material estudiado. Municipio de Ures, Sierra de Mazatán, A. Gutiérrez, E. Hernández, R. Maldonado y D. López, 10-09-2012, UES 10405.

Comentarios. Esta especie se caracteriza por su contexto dúplex, zonado, con depósitos resinosos, aunque difíciles de observar. Elementos de la cutícula fuertemente claviformes, generalmente enteros o con una protuberancia lateral. Moncalvo y Ryvarden (1997) y Ryvarden (2000) consideraron Ganoderma sessile como sinónimo de G. resinaceum Boud., no obstante Gottlieb y Wrigth (1999a) manejan estas especies como especies independientes, diferenciándolas microscópicamente por la ornamentación de las esporas y el tamaño de los pilares interparietales. Estos autores mencionaron que el holotipo de G. resinaceum se encontraba en mal estado, aceptando la posición de Murrill (1902), quien indicó esporas de 9-11 ×6-8 $\mu \mathrm{m}$, lo cual corresponde con el material estudiado. Por otra parte el tipo de G. sessile en NY parece tener problemas de identidad, como lo hicieron ver Moncalvo y Ryvarden (1997). Indudablemente G. sessile necesita de más estudios, sobre todo del tipo. Guzmán $(1977,1978)$ citó esta especie del centro de México, pero Mendoza et al. (2011) mencionaron que las colecciones de Guzmán corresponden a G. oerstedii. De esta manera, ésta es la primera cita de G. sessile para México. En Sonora se recolectó en un bosque de encino, en la base de Quercus sp.

Ganoderma sessiliforme Murrill, Bull. New York Bot. Gard. 8: 149 (1912) (Figura 1M-Ñ). Basidioma de 44-65 ×58-151 ×30 mm, anual, sésil a subestipitado. Píleo flabeliforme a conchado. Contexto claro sin bandas resinosas, homogéneo a relativamente homogéneo, azonado. Elementos de la cutícula de 31-53 × 7.5-14.3 $\mu \mathrm{m}$, claviformes, enteros a ocasionalmente con una protuberancia lateral, las cuales reaccionan con el reactivo de Melzer, tornándose negras inmediatamente. Basidiosporas de 8.8-11.1 (-11.5) ×6.2-7.2 (-7.6) $\mu \mathrm{m}, \mathrm{Q}=1.38-1.75$, elipsoides a oblongas, exosporio con pilares interparietales sublibres a libres. 


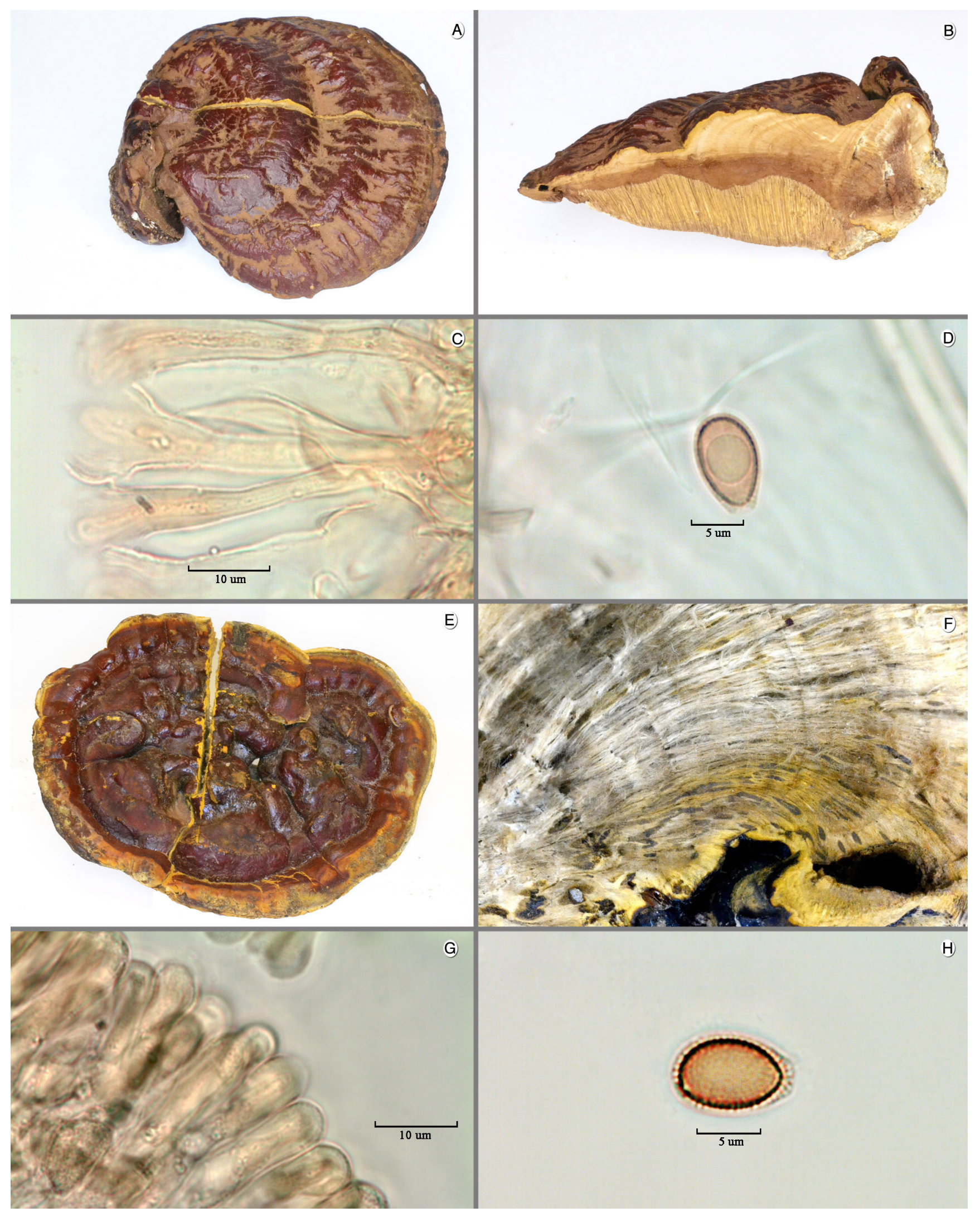

Figura 2. Ganoderma sessile y G. weberianum. G. sessile: A) basidioma, B) contexto, C) cutícula, D) Espora; G. weberianum: E) basidioma, F) contexto, G) cutícula, H) espora. 
Material estudiado. Municipio de Ures, Sierra de Mazatán, A. Gutiérrez, E. Hernández, R. Maldonado y D. López, 10-09-2012, UES 10407, 10408.

Comentarios. Las características distintivas de esta especie son su contexto claro, sin bandas resinosas, azonado, el cual es más grueso en la base, adelgazándose hacia el margen. Esta es una especie poco común. Fue registrada de Brasil (Torres-Torres et al., 2012), Argentina (Gottlieb y Wright, 1999a) y México (Raymundo et al., 2013). Esta es la segunda cita para Sonora, la primera fue por los últimos autores. Los ejemplares se recolectaron en bosque de encino sobre la base de Quercus sp.

Ganoderma weberianum (Bres. \& Henn. ex Sacc.) Steyaert, Persoonia 7(1): 79 (1972) (Figura 2E-H)

Basidioma de 45-200 × 30-185 × 20-52 mm, sésil a subestipitado cuando crece en el suelo sobre raíces, anual, solitario a gregario, de consistencia fibrosa a suberosa. Píleo semicircular a flabeliforme, aplanado a ligeramente convexo; superficie glabra, cubierta por una capa de laca brillante, con zonas opacas, abollada a irregular, ligeramente sulcada concéntricamente; marrón-rojizo (8F8) en la base o en el centro cuando subestipitada, amarillo-parduzco (5C7) a blanco-anaranjado (5A2) hacia el margen, el cual es estéril, obtuso a agudo, blanco-anaranjado $(5 \mathrm{~A} 2)$ en fresco, marrón (5F8) a negro cuando seco. Contexto de hasta $30 \mathrm{~mm}$ de grosor, relativamente homogéneo, zonado, con incrustaciones resinosas difíciles de observar, más conspicuas en la base, ausentes en algunos ejemplares, blanco anaranjado (5A2) a anaranjado pálido (5A3). Tubos de hasta $18 \mathrm{~mm}$ de largo, marrón-amarillento (5D5). Poros de 3-6 por mm, redondeados a angulados, concoloros con el margen, el cual es irregular, con zonas marrón cuando seco, de 122-287 $\mu \mathrm{m}$ diámetro; disepimento de 25-94 $\mu \mathrm{m}$. Sistema hifal dimítico. Hifas generativas de la trama del contexto no observadas; hifas esqueléticas de 2.1-9.7 $\mu \mathrm{m}$ diámetro, de pared gruesa a sólidas, sin septos, arboriformes y aciculiformes, amarillo pálido. Elementos de la cutícula de 33.8-50.6 × 6.5-12.2 $\mu \mathrm{m}$, cilíndricos a claviformes, con una o dos protuberancias o ramificaciones laterales a enteros, con gránulos en el ápice, amarillos; hifas generativas de 3.1-5.6 $\mu \mathrm{m}$ diámetro, pared delgada, con fíbulas, hialinas; hifas esqueléticas de 1-5.7 $\mu \mathrm{m}$ diámetro, pared gruesa a sólida, amarillo oro; hifas conectivas de 1-2.1 $\mu \mathrm{m}$ diámetro, ramificadas, pared gruesa a sólida, amarillenta a hialina. Basidiosporas de 7.4-10.3 × 5.3-6.7 (-7.1) $\mu \mathrm{m}, \mathrm{Q}=1.22-1.66$, generalmente elipsoides, algunas ampliamente elipsoides u oblongas, ápice truncado, perisporio perforado, exosporio con pilares interparietales sublibres a libres, delgados, marrón-amarillento. Basidios no observados.

Material estudiado. Municipio de Ures, Sierra de Mazatán, A. Gutiérrez, E. Hernández, R. Maldonado y D. López, 10-09-2012, UES 10415, 10417; C. Trujillo, E. Hernández, A. Gutiérrez y D. López, 27-09-2013, UES 10410, 10411, 10412, 10416.

Comentarios. Los caracteres distintivos de esta especie son el contexto zonado, claro, con incrustaciones resinosas en ocasiones difíciles de observar (ausentes en algunos de los ejemplares revisados), elementos de la cutícula cilíndricos a ligeramente claviformes, con incrustaciones apicales y esporas relativamente pequeñas. Pan y Dai (2001) mencionaron la presencia de clamidosporas en basidiomas de esta especie, lo cual no se observó en los ejemplares de Sonora. Este es el primer registro de G. weberianum para México. Recolectada en bosque de encino en la Isla de Montaña, en la base de un Quercus sp.

Distribución de Ganoderma en Sonora. Con base en los estudios previos (Esqueda et al., 2010; Raymundo et al., 2013) y en el actual, considerando todas las especies de Ganoderma registradas en Sonora, G. curtisii presenta la distribución conocida más amplia en el estado. Se encuentra en tres localidades: Reserva Forestal Nacional y Refugio de Fauna Silvestre Ajos-Bavispe (RFAB), Sierra de Mazatán y Sierra de San Javier; seguida por G. applanatum en la Sierra de Yécora y la zona urbana de Hermosillo; G. coffeatum (citada por Esqueda et al., 2010) en RFAB y Reserva de la Biosfera Sierra de Álamos-Río Cuchujaqui (RBAC); G. lucidum (Curtis) P. Karst. (citada por Esqueda et al., 2010) en RFAB y RBAC; G. sessiliforme (presente estudio y citada por Raymundo et al., 2013) en Sierra de Mazatán y Tuape. Con una localidad, G. adspersum (citada por Esqueda et al., 2010) en RFAB, G. lobatum en Sierra de Yécora, mientras que G. oerstedii, G. sessile y G. weberianum en la Sierra de Mazatán. De las diez especies de 
Ganoderma conocidas en Sonora, cinco se encuentran distribuidas en la Sierra de Mazatán, por lo que es una de las zonas con mayor diversidad de Ganoderma en la entidad.

\section{Discusión}

Algunas de las especies determinadas en este estudio presentan la medida de las basidiosporas diferente a lo que han registrado otros autores, como se ha comentado. Esto puede deberse a la gran variabilidad que existe o a la falta de más observaciones o malas determinaciones, lo cual podría aclararse con el estudio de los tipos. Ganoderma oerstedii se encontró en mezquital, en una zona con temperaturas mayores a $40{ }^{\circ} \mathrm{C}$, lo que muestra el grado de adaptación de esta especie. Las especies de la Sierra de Mazatán están en una isla ecológica rodeada por áreas xerófilas. Aquí se presentan distintos tipos de vegetación como mezquital en el pie de la montaña, seguido de matorral subtropical y bosque de encino en la parte alta (Piña-Páez et al., 2013). En esta área se encontró el $50 \%$ de las especies conocidas de Ganoderma para Sonora. Este comportamiento podría deberse a que los encinos están afectados por la epífita Tillandsia recurvata, la cual compite por la luz con el encino. Dicha condición está capitalizada por distintas especies de Ganoderma. Sería conveniente monitorear mejor la zona para entender los cambios ecológicos que ocurren. Por otra parte, es interesante observar que en Sonora se distribuyen cerca del $50 \%$ de las especies de Ganoderma conocidas en México, lo cual se puede deber a la intensidad de las exploraciones (p. ej. Esqueda et al., 2010) y a la diversidad de ecosistemas presentes en este estado.

Existen dudas sobre la distribución de Ganoderma lucidum en el Continente Americano y se especula que la especie está restringida a Europa, como lo han hecho ver Moncalvo et al. (1995) y Moncalvo y Ryvarden (1997). Los registros de G. lucidum en Sonora por Esqueda et al. (2010), deberán revisarse detalladamente para corroborar su identidad. Por otro lado, debido a la complejidad del género y a su amplia distribución en México, es necesario realizar una monografía a nivel nacional.

\section{Agradecimientos}

Los autores agradecen a Georgina Vargas por su apoyo técnico, así como a Alberto Jiménez, Carolina Trujillo y Rafael Maldonado por su apoyo en los muestreos. Asimismo a los revisores asignados de este manuscrito por sus valiosas observaciones.

\section{Literatura citada}

Bazzalo M.E. y Wright J.E. 1982. Survey of the Argentine species of the Ganoderma lucidum complex. Mycotaxon 16:293-325.

Esqueda M., Coronado M., Gutiérrez A., Valenzuela R., Chacón S., Gilbertson R.L., Herrera T., Lizárraga M., Moreno G., Pérez-Silva E. y Van Devender T.R. 2010. Hongos. En: Molina-Freaner E., Van Devender T.R. Eds., Diversidad Biológica de Sonora, pp. 189-205, Universidad Nacional Autónoma de México / Comisión Nacional para el Conocimiento y Uso de la Biodiversidad, México D.F

Furtado J.S. 1965. Ganoderma colossum and the status of Tomophagus. Mycologia 57:979-984.

Gilbertson R.L. y Ryvarden L. 1986. North American Polypores. Vol. 1. Abortiporus - Lindtneria. Fungiflora, Oslo.

Gottlieb A.M. y Wright J.E. 1999a. Taxonomy of Ganoderma from southern South America: subgenus Ganoderma. Mycological Research 103:661-673.

Gottlieb A.M. y Wright J.E. 1999b. Taxonomy of Ganoderma from southern South America: subgenus Elfvingia. Mycological Research 103:1289-1298.

Guzmán G. 1977. Identificación de los Hongos Comestibles, Venenosos, Alucinantes y Destructores de la Madera. Limusa, México D.F.

Guzmán G. 1978. Hongos. Limusa, México, D.F.

IFP (Index Fungorum Partnership). 2014. Index Fungorum. Disponible en: <http://www.indexfungorum. org/> (último acceso el 27 de febrero de 2014).

Kornerup A. y Wanscher J.H. 1978. Methuen handbook of color. Eyre Methuen, London.

Largent D.L., Johnson D. y Watling R. 1977. How to Identify Mushrooms to Genus III: Microscopic Features. Mad River Press, Eureka.

Mendoza G., Guzmán G., Ramírez-Guillén F., Luna M. y Trigos A. 2011. Ganoderma oerstedii (Fr.) Mu- 
Recibido:

28 de noviembre de 2014

Aceptado:

26 de enero de 2015 rrill (higher Basidiomycetes), a tree parasite species in Mexico: taxonomic description, rDNA study, and review of its medical applications. International Journal of Medicinal Mushrooms 13:545-552.

Moncalvo J.-M. y Ryvarden L. 1997. A Nomenclatural Study of the Ganodermataceae Donk. Synopsis Fungorum 11, Fungiflora, Oslo.

Moncalvo J.-M., Wang H.-F., Hseu R.-S. 1995. Gene phylogeny of the Ganoderma lucidum complex based on ribosomal DNA sequences. Mycological Research 99:1489-1499.

Murrill W.A. 1902. The Polyporaceae of North America I. The genus Ganoderma. Bulletin of the Torrey Botanical Club 29:599-608.

Pan H.-Y. y Dai Y.-C. 2001. Ganoderma weberianum newly recorded from mainland of China. Fungal Science 16:31-34.

Piña-Páez C., Esqueda M., Gutiérrez A. y González-Ríos H. 2013. Diversity of gasteroid fungi in the sierra de Mazatán, Sonora, México. The Southwestern Naturalist 58:351-356.

Raymundo T., Valenzuela R., Gutiérrez A., Coronado M.L. y Esqueda M. 2013. Agaricomycetes xilófagos de la planicie central del desierto Sonorense. Revista Mexicana de Biodiversidad 84:417-424.

Ryvarden L. 2000. Studies in Neotropical Polypores 2: A Preliminary key to Neotropical species of Ganoderma with a laccate pileus. Mycologia 92:180-191.

Steyaert R. L. 1980. Study of some Ganoderma species. Bulletin du Jardin botanique National de Belgique 50:135-186.

Torres-Torres M.G. y Guzmán-Dávalos L. 2005. Notas sobre la variación morfológica de Ganoderma curtisii (Ganodermatales, Ganodermataceae) en México. Revista Mexicana de Micología 21:39-47.

Torres-Torres M.G., Guzmán-Dávalos L. y de Mello Gugliotta A. 2012. Ganoderma in Brazil: known species and new records. Mycotaxon 121:93-132.

Zhao J.D. 1989. The Ganodermataceae in China. Bibliotheca Mycologica Band 132, J. Cramer, BerlinStuttgart. 